\title{
References
}

Flanagan, E. P., McKeon, A., Lennon, V. A., Boeve, B. F., Trenerry, M. R., Tan, K. M., ... \& Pittock, S. J. (2010, October). Autoimmune dementia: clinical course and predictors of immunotherapy response. In Mayo Clinic Proceedings (Vol. 85, No. 10, pp. 881-897). Elsevier.

McKeon, A., Lennon, V. A., \& Pittock, S. J. (2010). Immunotherapy-responsive dementias and encephalopathies. CONTINUUM: Lifelong Learning in Neurology, 16(2), 80-101.

Wotton, C. J., \& Goldacre, M. J. (2017). Associations between specific autoimmune diseases and subsequent dementia: retrospective record-linkage cohort study, UK. J Epidemiol Community Health, 71(6), 576-583.

\section{9 - PET@home: Research protocol for a toolkit to improve care for non-residential long term care clients owning pets}

\section{Author List}

Ruslan Leontjevas, Marie-José Enders-Slegers, Peter Reniers, Ine Declerq, Debby Gerritsen, Karin Hediger

\section{Background}

Over half of the households in The Netherlands have one or more pets. In elderly people, owning a pet is associated with a better quality of life and less loneliness, anxiety, depression and agitation. Many nonresidential long term care (LTC) clients rely on support of others to take care of their pets. However, that may place a significant burden on the social support network of the LTC client. Issues relevant to keeping pets are not explicitly incorporated in the Dutch Long-term Care Act. Many LTC organizations have no instruments for care workers, clients and their family (1) to consolidate the positive role of pets for clients' quality of life and (2) to address whether it is possible to keep the pets and to organize care accordingly.

\section{Research Objectives}

To help care workers, clients and their family to gain insight into the role of the pets in the clients' life and their social support network; to develop practical instruments that help making decisions about owning and caring for pets.

\section{Method}

PLAN: In months 0-16, a narrative systematic review will be conducted (STUDY 1.1) on the meaning of pets for elderly people in general. A qualitative STUDY 1.2 with LTC clients, their informal carers and care professionals will validate and further explore the topic. STUDY 1.3 and 1.4 develop and (cognitively) validate work cards for interviews of clients and relatives by care providers. In months 17-29, an Experience based co-design method (STUDIES 2.1-2.3) will be used to develop the PET@home toolkit. The method includes (1) discovery interviews (10 clients and their family), (2) focus groups with healthcare providers $(\mathrm{N}=2 \times 6)$; (3) focus groups with 6-8 clients and informal and professional carers. In STUDY 3.1, potential users will pre-test the Toolkit. In months 30-34, a process evaluation (STUDY 3.2) is performed in 10-15 clients. A dissemination and an implementation plan will be developed.

\section{Conclusions}

The project will result in an innovative PET@home toolkit that will help to assess the pets role in the clients' quality of life and support network, and will help making decisions about owning and caring for pets. 